Relations industrielles

Industrial Relations

\title{
Adams, Roy J., ed. Comparative Industrial Relations: Contemporary Research and Theory
}

\section{Guy Bellemare}

Volume 48, numéro 3, 1993

URI : https://id.erudit.org/iderudit/050879ar

DOI : https://doi.org/10.7202/050879ar

Aller au sommaire du numéro

Éditeur(s)

Département des relations industrielles de l'Université Laval

ISSN

0034-379X (imprimé)

1703-8138 (numérique)

Découvrir la revue

Citer ce compte rendu

Bellemare, G. (1993). Compte rendu de [Adams, Roy J., ed. Comparative Industrial Relations: Contemporary Research and Theory]. Relations industrielles / Industrial Relations, 48(3), 566-567.

https://doi.org/10.7202/050879ar

Tous droits réservés @ C Département des relations industrielles de l'Université Laval, 1993
Ce document est protégé par la loi sur le droit d'auteur. L'utilisation des services d'Érudit (y compris la reproduction) est assujettie à sa politique d'utilisation que vous pouvez consulter en ligne.

https://apropos.erudit.org/fr/usagers/politique-dutilisation/ 


\section{RECENSIONS BOOK REVIEWS}

Comparative Industrial Relations: Contemporary Research and Theory, Roy J. Adams, ed., London, England, Harper Collins, 1991, 145 p., ISBN 0-04-445966-1 and ISBN 0-04-445967-X (pbk).

Ce volume réunit les huit contributions présentées dans le cadre d'un atelier portant sur la recherche comparative en relations industrielles lors du $8^{\circ}$ Congrès mondial de l'Association internationale des relations professionnelles tenu en 1989. Cet atelier était présidé par Roy J. Adams qui s'est chargé de la publication des textes et de l'introduction du volume. Celui-ci est construit autour du modèle du système des relations industrielles (SRI) de Dunlop et il examine, d'une part, la théorie et le SRI et, d'autre part, l'évolution du rôle de ses trois acteurs.

Les articles de Meltz et de Barbash sont consacrés à la réhabilitation des théories de Dunlop sur le SRI et de Commons sur les relations du travail. Meltz rejette les critiques classiques de la théorie de Dunlop (son statisme, son postulat d'idéologie commune des acteurs, etc.) et suggère que ce sont plutôt les chercheurs qui ont failli à la tâche : "Thus for Dunlop the purpose of the theory is to be able to examine specific developments in the work place and to test hypotheses on why they are occuring. In general, this seems not to have been done ". Barbash présente un portait de Commons, père fondateur des relations industrielles, avec les Weeb, Marx Taylor et Mayo. Barbash veut rappeler aux acteurs du champ disciplinaire des relations industrielles le sens de leurs origines et de leurs valeurs. Commons voulait améliorer la condition ouvrière à l'aide de la négociation collective, de la législation et de la formation des gestionnaires. Barbash le présente non seulement comme un théoricien (théorie de l'efficacité et de l'équité) mais aussi comme le créateur d'une méthode, celle de l'observation participante, par ses tâches de chercheur, de médiateur, d'administrateur, etc. Adams rappelle que même si la théorie et le champ disciplinaire des relations industrielles se sont développés surtout dans les pays anglo-saxons industrialisés et à économie de marché, ils tendent à s'étendre maintenant aux pays de l'Est et en voie de développement. Il en prend pour exemple les articles de Héthy sur la construction du système de relations industrielles et l'évolution des relations tripartites de relations du travail dans les pays de l'Est et ceux de Fashoyin sur les développements récents de la pratique des relations industrielles dans les pays en développement où la négociation collective indépendante progresse.

Les quatre autres articles analysent l'évolution du rôle et des relations des trois acteurs du SRI au cours de la dernière décennie. Price effectue une analyse comparative de la diminution du taux de syndicalisation inspirée de l'approche de Dunlop et conclut que les seuls facteurs économiques ne peuvent expliquer l'évolution différenciée du taux de syndicalisation dans les pays capitalistes avancés durant les périodes de changement important. Ce sont alors plutôt des variables institutionnelles qui doivent être retenues 
(cadre légal, politiques syndicales et patronales, etc.). Plowman analyse justement ces changements aux politiques patronales alors que Thompson en montre les conséquences sur la négociation collective. Les employeurs ont cherché à transformer de façon radicale leur gestion du personnel, à éliminer la présence syndicale dans certains cas (ÉtatsUnis) et à décentraliser et à rendre plus flexible les relations du travail dans la plupart des pays capitalistes avancés. Pour sa part, Keller examine les politiques étatiques de différents pays et conclut que les rigidités généralement associées aux régulations étatiques n'ont pas pour effet unidirectionnel de réduire la productivité des entreprises et économies nationales comme le supposent les thèses néo-libérales.

Ce volume constitue une bonne source de documentation pour les étudiants inscrits à un cours de relations industrielles comparées. Mais il laissera songeur ceux qui se préoccupent de l'avenir des relations industrielles comme champ de la pratique sociale et comme champ scientifique. En effet, au plan de la pratique, alors que les systèmes nationaux de relations industrielles sont en transformation radicale, que le syndicalisme décline dans de nombreux pays, que les modes de gestion du personnel se transforment, etc., le volume traite surtout des thèmes traditionnels en relations industrielles (par exemple, l'étude du taux de syndicalisation, le rôle de l'État et la négociation collective). Plowman et Thompson font tout de même mention d'approches différentes de l'étude des relations industrielles avec la notion de labour process ou des solutions de rechange à la négociation collective. Au plan du champ scientifique, alors que le paradigme théorique dunloppien d'orientation néo-positiviste sur lequel se sont construites les relations industrielles connaît une crise et qu'on assiste à une multiplication des paradigmes et à l'apparition des théories post-structuralistes en relations industrielles, que l'avenir même des départements de relations industrielles est remis en question aux États-Unis, en Australie et ailleurs, on demeure étonné à la lecture du volume de l'absence de remises en question telles, la réhabilitation de la théorie dunloppienne, la ré-explication de Commons: science as usual, véritable cas de "science normale " au sens de Kuhn (La structure des révolutions scientifiques). Est-ce le champ des relations industrielles qui refuse de se remettre en question ou est-ce le reflet du choix éditorial? Une réponse positive à la première question serait plus inquiétante qu'une réponse positive à la deuxième. Elle signifirait que le champ scientifique des relations industrielles est décroché des relations industrielles contemporaines et des mouvements de la science moderne (montée des épistémologies anti-positiviste constructiviste et interprétative), alors que la seconde signifierait que l'éditeur n'atteint pas totalement son objectif de présenter les théories et recherches comparatives contemporaines.

Guy Bellemare

Université du Québec à Hull

Masters to Managers: Historical and Comparative Perspectives on American Employers, Sanford P. Jacoby, ed., New York, Oxford, Columbia University Press, 1991, 249 p., ISBN 0-231-06802-6

This book represents a fascinating treatment of an important subject. The industrial relations literature is replete with historical studies of trade unions and their 